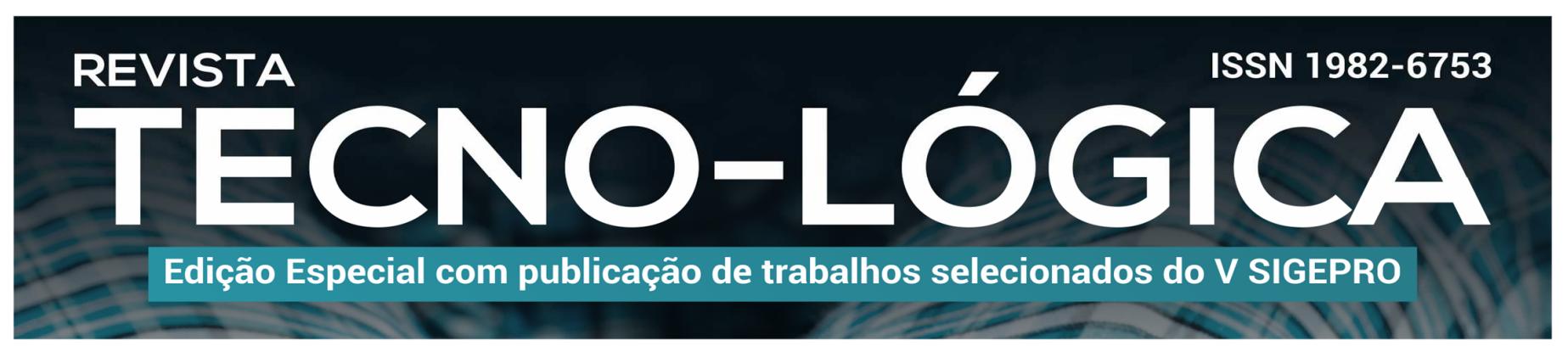

\title{
APLICAÇÃO DE PROGRAMAÇÃO DINÂMICA PARA OTIMIZAÇÃO DE UM LAYOUT DE PRODUÇÃO: UM ESTUDO DE CASO
}

Amanda Dalla Rosa Monegat ${ }^{1}{ }^{*}$, Mateus Müller Franco ${ }^{1}$, Bruna Caroline Orlandin ${ }^{1}$, Leandro Luis Corso ${ }^{1}$

${ }^{1}$ Curso de Engenharia de Produção, Universidade de Caxias do Sul, CEP 95070-560, Caxias do Sul/RS, Brasil

*E-mail: $\underline{\text { adrmonegat@ucs.br }}$

Recebido em: $17 / 09 / 2020$

Aceito em: 06/11/2020

DOI: 10.17058/tecnolog.v2i0.15750

\section{RESUMO}

Frequentemente, máquinas estão localizadas em locais não planejados, criando fluxos de materiais com baixa eficiência de distribuição. Este artigo apresenta um estudo de caso de programação dinâmica determinística aplicada à análise de modificação de layout de uma planta industrial, a fim de aperfeiçoar o deslocamento interno para reduzir os custos de movimentação. Para isso, foram identificados os possíveis posicionamentos para as três máquinas consideradas neste trabalho e as distâncias de deslocamento. Como resultado, obteve-se um layout otimizado em relação aos cenários possíveis, com redução de deslocamento em 10\%. Sendo assim, tornase evidente a contribuição da programação dinâmica como ferramenta de suporte para processos de modificação de layout.

Palavras-chave: Pesquisa operacional. Programação matemática. Programação dinâmica. Determinística. Layout.

\section{Introdução}

Um dos grandes desafios atuais para as indústrias, em resposta às mudanças de requisitos e oportunidades, é a necessidade permanente de rápida reconfiguração das suas plantas fabris [1]. Segundo os autores pesquisados, a alta variedade de produtos fabricados simultaneamente e a vida útil cada vez menor desses produtos fazem com que a necessidade de ajustes nos layouts industriais cresça significativamente. De acordo com Vitayasak, Pongcharoen e Hicks [2], a decisão de alterar um layout deve levar em consideração o seu rearranjo e a distância total percorrida pelos materiais, sendo isso um proxy comumente usado para medir a eficiência dos layouts. Frequentemente, máquinas estão localizadas em locais não planejados, criando fluxos de materiais difíceis que diminuem a eficiência [1]. Neste contexto, Cellin [3] afirma que métodos de otimização entram como uma importante ferramenta para auxiliar esse processo.

Para Cardoso [4], a Programação Dinâmica (PD) é uma poderosa técnica de otimização de sistemas dinâmicos, fundamentada nos trabalhos publicados por Bellman, assim como os posteriores, mostrando a importância do tema e suas numerosas 


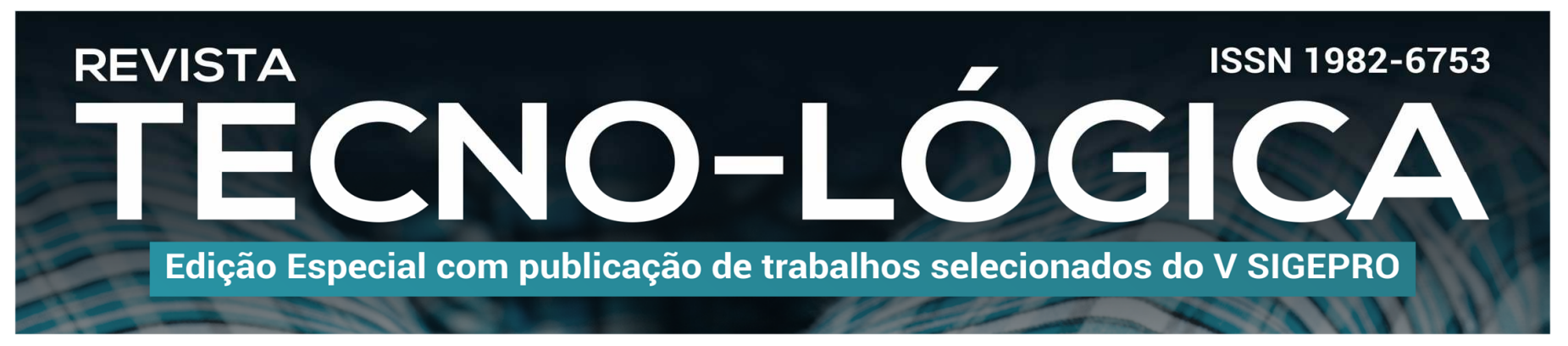

aplicações. Como complemento, Arenales et al. [5] destacam que a principal característica da PD consiste na decomposição do problema original em uma sequência de problemas menores e mais simples de serem resolvidos. Assim, é possível a aplicação na resolução de diversos problemas de otimização.

Isso posto, encontram-se múltiplos estudos na bibliografia envolvendo programação dinâmica em diferentes áreas de atuação, possibilitando a análise de diversas aplicações. Nesse contexto, algumas publicações podem ser destacadas, como Silva e Gualda [6], na alocação de recursos de transporte de cargas; Rauppi, Gibon e Beuren [7], com a otimização de recursos escassos na contabilidade de custos; Boomen, Berg e Wolfert [8], na otimização econômica de manutenção e substituição de ativos; Ke, Zhang e Zheng [9], aplicando preços dinâmicos no gerenciamento de receita de redes; e Cechin et al. [10], com a redução dos custos na compra de vacinas de um hospital.

Dessa maneira, este artigo possui como objetivo principal apresentar um estudo de caso de PD, propondo a modificação do layout fabril de uma empresa. Sendo assim, tem em vista encontrar o melhor posicionamento das máquinas que fazem parte deste estudo, de forma a minimizar o deslocamento da empilhadeira que abastece os equipamentos. Dessa forma, este trabalho está estruturado em cinco seções, propiciando a aplicação teóricoprática do conteúdo adotado. Elas são denominadas, respectivamente, como introdução, referencial bibliográfico, método, resultados e discussões, considerações finais e referências.

\section{Referencial Teórico}

Nesta etapa apresentam-se os principais conceitos necessários para o desenvolvimento deste trabalho, alinhados aos objetivos da pesquisa.

\subsection{Programação Dinâmica}

A PD foi criada por Richard Bellman, em 1957, com a publicação livro intitulado Dynamic Programming [11]. Para Hillier e Lieberman [12], a PD é uma técnica matemática útil para tomar uma sequência de decisões que estão mutuamente relacionadas. Com ela, é possível obter um procedimento sistemático na determinação de decisões ótimas. De acordo com os autores, não há uma formulação matemática padrão para os problemas a serem tratados e modelados com PD. Sendo assim, as equações devem ser desenvolvidas para cada situação, buscando a resolução dos problemas.

Segundo Hillier e Lieberman [12], os problemas de PD possuem características importantes de serem destacadas. São elas:

a) O problema pode ser dividido em estágios;

b) Cada estágio possui um número de estados associados ao seu início;

c) Busca-se transformar o estado atual em um estado associado ao início do estágio seguinte;

d) O procedimento de resolução é desenhado para encontrar uma política ótima para o problema como um todo;

e) O princípio da otimalidade é baseado no fato de que a decisão ótima depende somente do estado atual, e não de como se chegou até lá. 


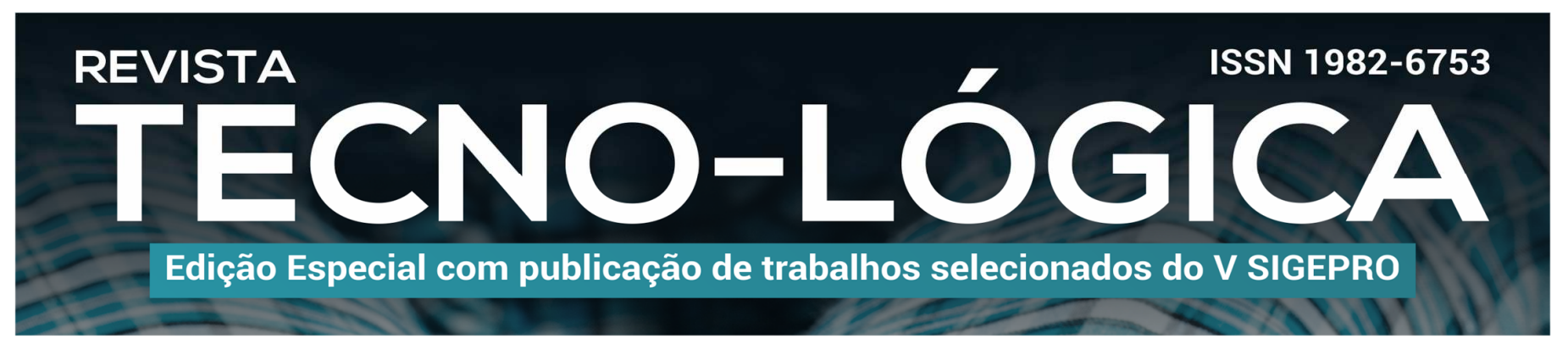

Para Cechin et al. [10], o objetivo principal está em encontrar a melhor solução para todas as instâncias dos estados. Dessa maneira, com a combinação das respostas obtidas, é possível encontrar o resultado do processo original. Como complemento, Arenales et al. [5] classificam os modelos de PD como determinístico e probabilístico. No caso da PD determinística, conforme Hillier e Lieberman [12, p. 411], “o estado no estágio seguinte é determinado completamente pelo estado e decisão sobre a política a ser adotada no estágio atual". Nela, o período de otimização é caracterizado como finito e as variáveis de estado são discretas. Já em modelos probabilísticos, os autores explicam que existe uma distribuição de probabilidade envolvida com o estado seguinte. Ainda para Hillier e Liberman [12, p. 431], "o estado no estágio seguinte não é completamente determinado pelo estado e pela decisão sobre a política a ser adotada no estágio atual”. Com relação ao tempo, a PD é dividida em contínua e discreta.

Conforme Cechin et al. [10] e Colin [11], um modelo de PD é composto por um estágio inicial t qualquer, um estado de entrada $\mathrm{S}_{0}$, estados de saída $\mathrm{S}_{\mathrm{t}}$, variável de decisão $\mathrm{X}_{\mathrm{t}}$, um custo do estágio $\mathrm{f}_{\mathrm{t}}\left(\mathrm{e}_{\mathrm{t}-1}, \mathrm{x}_{\mathrm{t}}\right)$, e a transformação $\varphi_{\mathrm{t}}$ do estágio t. $\mathrm{O}$ custo $\mathrm{f}_{\mathrm{t}}\left(\mathrm{e}_{\mathrm{t}-1}\right.$, $\mathrm{x}_{\mathrm{t}}$ ) mede a eficiência da transformação das entradas em saídas, e $\varphi_{\mathrm{t}}$ expressa as saídas como uma função das entradas, ou seja, $\mathrm{S}_{\mathrm{t}}=$ $\varphi_{\mathrm{t}}\left(\mathrm{S}_{\mathrm{t}-1}, \mathrm{x}_{\mathrm{t}}\right)$. As equações de continuidade de fluxo e o cálculo do custo são demonstradas respectivamente nas Equações 1 e 2 [11].

$e_{t}=e_{t-1}+x_{t}-d_{t}$

máx. ou min. $f_{t}\left(e_{t-1}, x_{t}\right)=$ máx. ou min $\left[\left(\right.\right.$ custo do estado $\left.S_{t-1}\right)+$ $\left(\right.$ custo viável de decisão $\left.x_{t}\right)+\left(\right.$ custo de estado $\left.\left.S_{t+1}\right)\right]$

\section{Método}

A pesquisa realizada tem como objeto de estudo uma planta industrial composta por três máquinas. Este trabalho visa estabelecer o melhor roteiro de processo por meio do reposicionamento de layout. Ou seja, o objetivo é encontrar o melhor posicionamento das máquinas, dentre as opções possíveis, que minimizem a movimentação interna dos componentes entre os processos.

Dessa forma, o presente trabalho apresenta um estudo de caso de natureza aplicada e abordagem quantitativa [13], pertencente à área de Pesquisa Operacional e à subárea de Programação Matemática. Para atingir o objetivo proposto, este trabalho foi dividido em três etapas que representam os objetivos específicos, conforme a Figura 1.

\begin{tabular}{|c|c|c|}
\hline Etapa 1 & Etapa 2 & Etapa 3 \\
\hline $\begin{array}{l}\text { - Mapeamento de } \\
\text { cenários e } \\
\text { restrições; }\end{array}$ & $\begin{array}{l}\text { Desenvolvimento } \\
\text { do modelo de } \\
\text { PD; }\end{array}$ & $\begin{array}{l}\text { - Resolução do } \\
\text { modelo e } \\
\text { interpretação dos } \\
\text { resultados; }\end{array}$ \\
\hline
\end{tabular}

Figura 1 - Etapas da metodologia.

$\mathrm{Na}$ primeira etapa, foram realizados o mapeamento dos cenários para o novo layout e a identificação das restrições relacionadas. Para isso, a distância entre as possíveis posições dos equipamentos foram analisadas. Nessa fase, buscou-se relacionar as seguintes informações: posições das máquinas, distâncias entre elas, restrições de layout e restrições de processo.

$\mathrm{Na}$ etapa 2, as informações previamente listadas foram relacionadas aos parâmetros da programação dinâmica e foi construído o modelo de PD representado por um esquema sequencial. Os resultados obtidos nessa etapa foram utilizados como base para solução do modelo na etapa seguinte. 


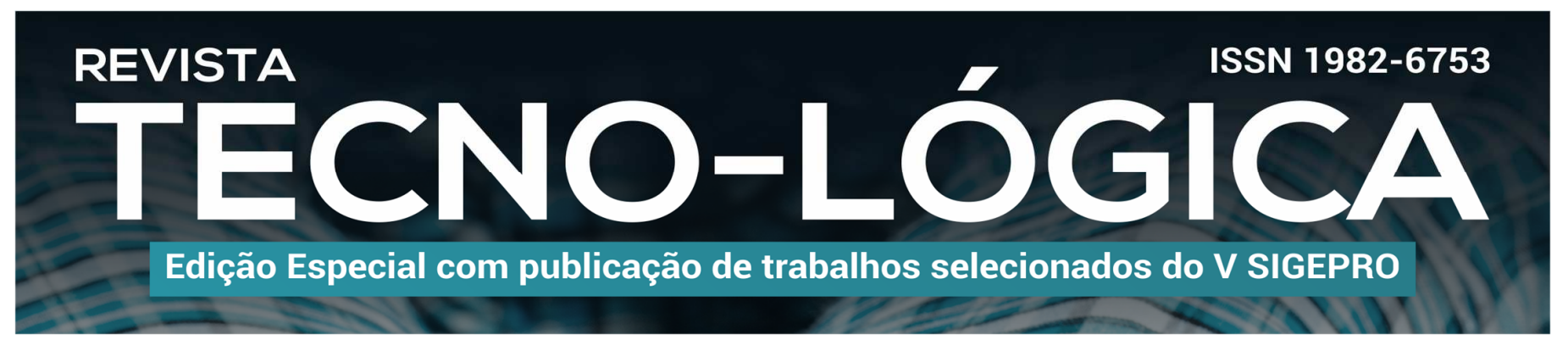

Na Etapa 3, verificou-se que em análises de algoritmos desenvolvidos por PD e pela praticidade comprovada por testes realizados na década de 1980, o cálculo ocorre de trás para frente, assim como implementado por Diban et al. [14] em seu trabalho. Portanto, para o processo em questão, o cálculo iniciou-se pelo posicionamento da máquina 3 em relação ao ponto de saída do produto acabado, depois foi calculado o melhor posicionamento para a máquina 2 , e finalizando pela definição da posição da máquina 1 .

\section{Resultados e discussões}

Nesta seção apresentam-se o desenvolvimento de cada uma das etapas deste trabalho e os resultados obtidos.

\subsection{Mapeamento de cenários e restrições}

Seguindo a metodologia apresentada na seção anterior, na etapa 1 , foram identificados os possíveis posicionamentos para os três recursos considerados neste trabalho e as distâncias de deslocamento, a fim de encontrar a solução ótima que minimize a movimentação a partir da entrada da matéria-prima até a saída do produto final. Como resultado para o mapeamento, existem três possíveis posições para as máquinas 1 e 2 , e duas possíveis posições para a máquina 3, criando diferentes cenários para o novo layout. No entanto, há uma restrição quanto à localização da máquina 1. Se esta for alocada na segunda opção de posicionamento possível, a localização da máquina 2 reduz de três posições possíveis para uma.

\subsection{Desenvolvimento do modelo de PD}

TECNO-LÓGICA, Santa Cruz do Sul, v. 24, n. nesp, p. 317-323, jul./dez. 2020
Na etapa 2 foi construído o modelo de PD demonstrando os cenários conforme apresentado na Figura 2, no qual as distâncias estão em metros.

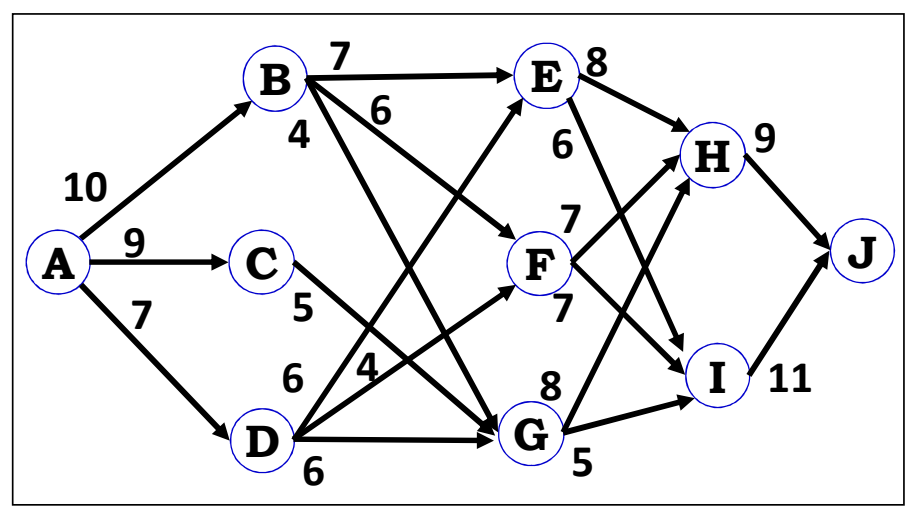

Figura 2 - Modelo de PD representando o problema de layout.

A Figura 2 apresenta as possíveis posições para as máquinas, sendo que o ponto A é o ponto de entrada da matériaprima, os pontos $\mathrm{B}, \mathrm{C}$ e D são as possíveis posições para a máquina 1, E, F e G para a máquina 2, H ou I para a máquina 3 e J é o ponto de saída do produto final. Além disso, os números demonstrados na Figura 2 representam as distâncias entre cada ponto. Observando o modelo estruturado, é entendido que o ponto A é o estado de entrada e 0 , J é o estado de saída final et, as posições dos equipamentos são variáveis de decisão xt, e que as distâncias partindo de $A$ até $J$ são $f_{t}\left(e_{t-1}, x_{t}\right)$.

\subsection{Resolução do modelo e interpretação dos resultados}

Para este do estudo de caso, o modelo de PD foi dividido em quatro estágios. O estágio um parte do ponto $\mathrm{A}$, o segundo estágio parte de B, C ou D, o terceiro estágio sai de E, F ou G, e o quarto estágio de $\mathrm{H}$ ou I. Dessa forma, o estágio inicial para resolução do modelo foi o estágio 4, considerando a metodologia 


\section{REVISTA}

\section{Edição Especial com publicação de trabalhos selecionados do V SIGEPRO}

de trás para frente. O Quadro 1 apresenta os resultados obtidos para análise do posicionamento da máquina $3 \mathrm{em} \mathrm{H}$ ou I.

Quadro 1 - Resolução do modelo de PD estágio 4

\begin{tabular}{|c|c|c|}
\hline $\mathrm{n}=4$ & $\mathrm{f} 4(\mathrm{~s}, \mathrm{x} 4)$ & \\
\hline $\mathrm{f} \backslash \mathrm{x} 4$ & $\mathrm{~J}$ & $\mathrm{x} 4^{*}$ \\
\hline $\mathrm{H}$ & 9 & $\mathrm{~J}$ \\
\hline $\mathrm{I}$ & 11 & $\mathrm{~J}$ \\
\hline
\end{tabular}

Pelo Quadro 1, constata-se que a distância de deslocamento entre a máquina 3 até $\mathbf{J}$, sendo esta a variável de decisão neste estágio, é 9, se o recurso estiver localizado em $\mathrm{H}$, ou 11, se estiver localizado em I. O Quadro 2 apresenta os resultados obtidos para o estágio 3 .

Quadro 2 - Resolução do modelo de PD estágio 3.

\begin{tabular}{|c|c|c|c|c|}
\hline $\mathrm{n}=3$ & \multicolumn{2}{|c|}{$\mathrm{f} 3(\mathrm{~s}, \mathrm{x} 3)$} & & \\
\hline $\mathrm{s} \backslash \mathrm{x} 3$ & $\mathrm{H}$ & $\mathrm{I}$ & $\mathrm{f3} *(\mathrm{~s})$ & $\mathrm{x} 3^{*}$ \\
\hline $\mathrm{E}$ & 17 & 17 & 17 & $\mathrm{H}$ ou I \\
\hline $\mathrm{F}$ & 16 & 18 & 16 & $\mathrm{H}$ \\
\hline $\mathrm{G}$ & 17 & 16 & 16 & $\mathrm{I}$ \\
\hline
\end{tabular}

Para o estágio 3, é possível partir de E, F ou G para $\mathrm{H}$ ou I. Conforme evidenciados pela figura, considerando que a máquina 2 seja posicionada em E, a máquina 3 pode ser posicionada tanto em $\mathrm{H}$ quanto em $\mathrm{I}$, pois o deslocamento até J é o mesmo. Já considerando que o recurso 2 esteja localizado em F, a melhor opção para o recurso 3 é estar posicionado em $\mathrm{H}$. Para a localização em G, o melhor local para a máquina 3 é em I. O Quadro 3 apresenta os resultados obtidos para o estágio 2.
Quadro 3 - Resolução do modelo de PD estágio 2.

\begin{tabular}{|c|c|c|c|c|c|}
\hline $\mathrm{n}=2$ & \multicolumn{3}{|c|}{$\mathrm{f} 2(\mathrm{~s}, \mathrm{x} 2)$} & & \\
\hline $\mathrm{s} \backslash \mathrm{x} 2$ & $\mathrm{E}$ & $\mathrm{F}$ & $\mathrm{G}$ & $\mathrm{f} 2 *(\mathrm{~s})$ & $\mathrm{x} 2 *$ \\
\hline $\mathrm{B}$ & 24 & 22 & 20 & 20 & $\mathrm{G}$ \\
\hline $\mathrm{C}$ & - & - & 21 & 21 & $\mathrm{G}$ \\
\hline $\mathrm{D}$ & 23 & 20 & 22 & 20 & $\mathrm{~F}$ \\
\hline
\end{tabular}

No estágio 2, é possível ir até J partindo de B, C ou D para E, F ou G. Desta forma, considerando que a máquina 1 seja posicionada em $\mathrm{B}$, o melhor posicionamento para a máquina 2 é $\mathrm{G}$ e por consequência, conforme descrito no estágio 3, a máquina 3 deverá ser posicionada em I. Com o equipamento 1 localizado em C, o melhor posicionamento para os equipamentos 2 e 3 são respectivamente em $\mathrm{G}$ e I. E para o recurso 1 posicionado $\mathrm{D}$, a posição dos recursos 2 e 3 que minimizam o deslocamento é em $F$ e H. O Quadro 4 apresenta os resultados obtidos para o estágio 1.

Quadro 4 - Resolução do modelo de PD estágio 1.

\begin{tabular}{|c|c|c|c|c|c|}
\hline $\mathrm{n}=1$ & \multicolumn{3}{|c|}{$\mathrm{f} 1(\mathrm{~s}, \mathrm{x} 1)$} & & \\
\hline $\mathrm{s} \backslash \mathrm{x} 1$ & $\mathrm{~B}$ & $\mathrm{C}$ & $\mathrm{D}$ & $\mathrm{f} 1 *(\mathrm{~s})$ & $\mathrm{x} 1 *$ \\
\hline $\mathrm{A}$ & 30 & 30 & 27 & 27 & $\mathrm{D}$ \\
\hline
\end{tabular}

No estágio 1 é possível partir apenas do ponto A indo para B, C ou D. A partir do ponto inicial, ou seja, o ponto de entrada de matéria-prima, se a máquina 1 for posicionada em $\mathrm{B}$ ou em $\mathrm{C}$ o deslocamento total até a saída do produto final será 30 metros. No entanto, se a máquina 1 for posicionada em D se obterá um deslocamento total de 27 metros, sendo este um valor menor do que o obtido para as duas possibilidades anteriores.

Assim, a partir dos resultados obtidos em cada estágio pela aplicação da programação dinâmica, o layout que otimiza o roteiro de processo da fábrica por meio da redução do 


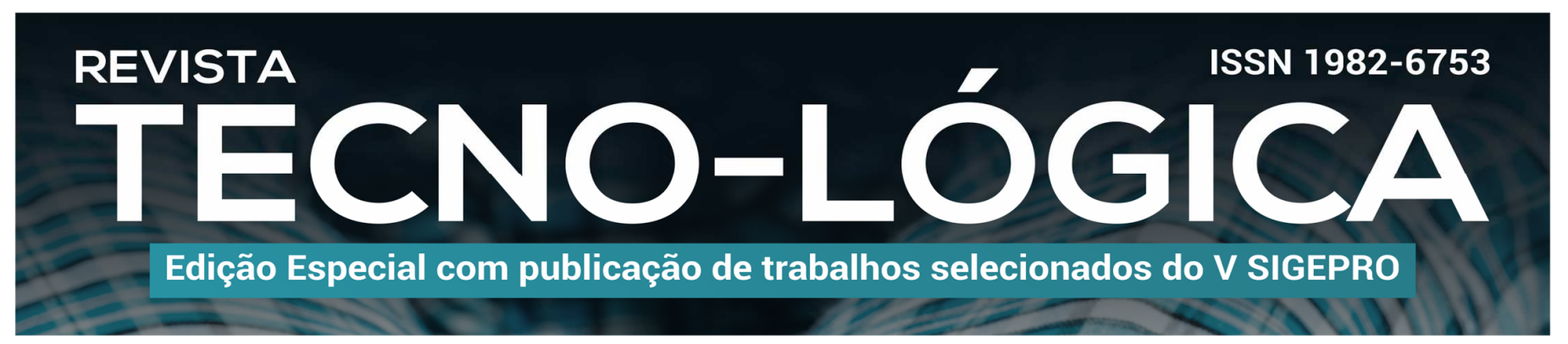

descolamento é A - D - F - H - J. Ou seja, partindo do ponto A (entrada de matéria-prima), a máquina 1 deverá ser posicionada no ponto $\mathrm{D}$, a máquina 2 no ponto $\mathrm{F}$ e a máquina 3 no ponto $\mathrm{H}$. Por fim, a Figura 3 apresenta o layout ideal obtido com resultado da aplicação do método.

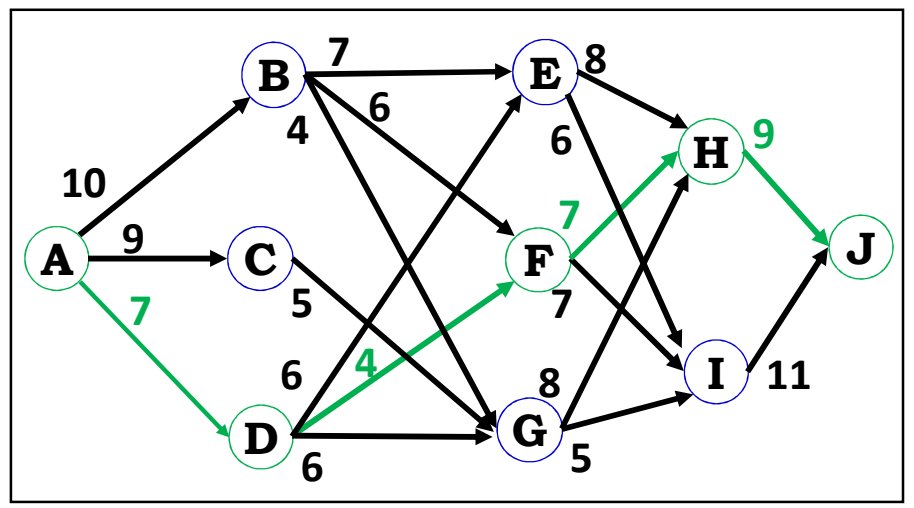

Figura 3 - Representação do layout ótimo obtido por PD.

\section{Conclusões}

Este trabalho teve como principal objetivo propor a modificação do layout fabril de uma empresa por meio da utilização de PD, tendo em vista encontrar o melhor posicionamento das máquinas, de forma a minimizar $o$ deslocamento percorrido pela empilhadeira utilizada no processo. A partir disso, ficou evidente a contribuição da utilização de um modelo matemático para auxiliar em processos de modificação de layout.

Dentre os arranjos possíveis, dois deles gerariam como resultado 30 metros de deslocamento de A até J, e um deles, 27 metros. Como resultado, obteve-se a minimização de $10 \%$ em deslocamento para o layout ótimo em relação aos outros cenários possíveis, o que corresponde a três metros. Sendo assim, o objetivo foi alcançado, uma vez que se obteve uma configuração para a planta fabril capaz de reduzir as movimentações internas e os custos envolvidos.

Os resultados foram satisfatórios, visto que modificações de layout implicam diretamente os sistemas de produção e os indicadores industriais, necessitando atender às prioridades competitivas das organizações. Como principal limitação deste estudo, pode-se citar a disponibilidade de apenas uma variável para análise. Dessa forma, a otimização das movimentações internas e a minimização dos custos são limitadas a este fator.

Como trabalhos futuros, sugere-se a aplicação de simulação no objeto deste estudo, por meio de um software computacional, buscando avaliar e comparar as métricas a serem encontradas com os resultados obtidos neste artigo. Dessa maneira, com a simulação computacional, será possível diminuir a imprevisibilidade e os custos, assim como incluir todas as variáveis envolvidas no processo produtivo. Ainda, a aplicação resultante desta pesquisa, com a utilização de $\mathrm{PD}$, poderá ser replicada em outras células da organização em questão, objetivando minimizar os tempos de deslocamento e os custos envolvidos com tais processos.

\section{DYNAMIC PROGRAMMING APPLICATION FOR OPTIMIZING AN INDUSTRIAL PRODUCTION}

\section{LAYOUT: A CASE STUDY}

ABSTRACT: Machines are often placed in unplanned locations, which causes low distribution efficiency regarding material flow. This paper is a case study of deterministic dynamic programming, applied to the layout modification analysis of an industrial plant, developed in order to optimize its internal displacement needs and 


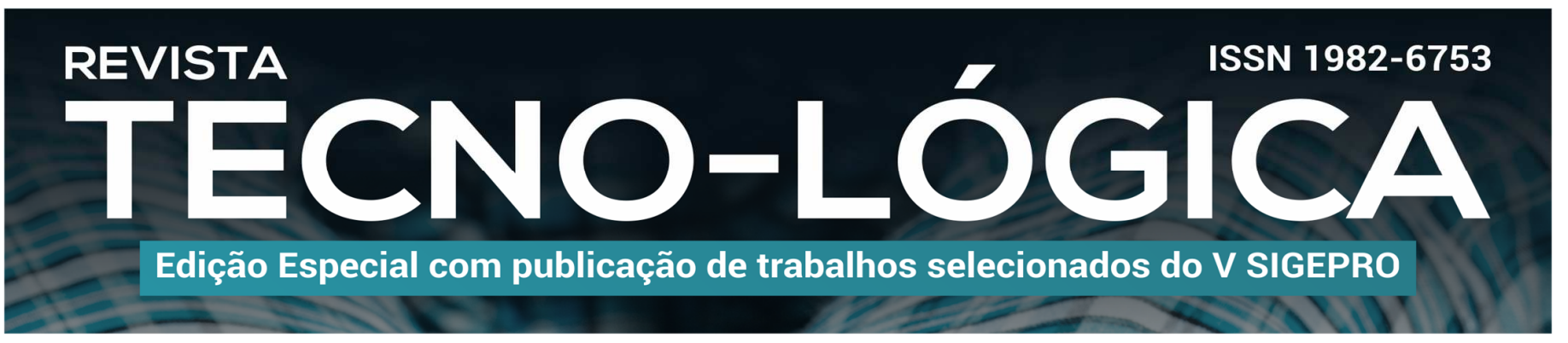

reduce handling costs. Thus, we identified the possible positionings for the three machines considered in this study and their displacement distances. As a result, an optimized layout was obtained considering the possible scenarios, with displacement reduction by $10 \%$. Thus, the contribution of dynamic programming as a support tool for layout modification processes becomes evident with this case study.

Keywords: Operational research. Mathematical programming. Dynamic programming. Deterministic. Layout.

\section{Referências}

[1] AZEVEDO, M. M.; CRISPIM, J. A.; SOUSA, J. P. de.; A dynamic multiobjective model for designing machine layouts. Ifac-papersonline, Vol. 52, $\mathrm{n}^{\circ} .13, \mathrm{p} .1896-1901,2019$.

[2] VITAYASAK, S.; PONGCHAROEN, P.; HICKS, C.; Robust machine layout design under dynamic environment: Dynamic customer demand and machine maintenance. Expert Systems With Applications: X, Vol. 3, p.100015, 2019.

[3] CELLIN, B. de P. Métodos para resolução eficiente de problemas de layout. 2017. 73 f. Dissertação (Programa de Pós-graduação em Informática - Mestrado) Universidade Federal do Espírito Santo, Vitória. 2017.

[4] CARDOSO, R. T. N. Algoritmos para programação dinâmica baseados em famílias invariantes. 2005. 115 f. Dissertação (Curso de Matemática, Departamento de Matemática - Mestrado) - Universidade Federal de Minas Gerais, Belo Horizonte. 2005

[5] ARENALES, M. et al. Pesquisa Operacional para cursos de Engenharia. 6. Ed. Rio de Janeiro: Elsevier, 2007.

[6] SILVA, A. M. L.; GUALDA, N. D. F.; Programação dinâmica aplicada à alocação de recursos no transporte de cargas. Transportes, Vol. 16, nº. 2, p 41-47, 2008 .

[7] RAUPP, F. M.; GIBBON, A. R. de O.; BEUREN, I. M.; O uso da programação dinâmica pela contabilidade de custos na otimização de recursos escassos. Contabilidade Vista \& Revista, Vol. 15, nº. 1, p.73-89, 2009..
[8] BOOMEN, M. van D.; BERG, P. L. van D.; WOLFERT, A. R. M.; A dynamic programming approach for economic optimisation of lifetime-extending maintenance, renovation, and replacement of public infrastructure assets under differential inflation. Structure and Infrastructure Engineering, Vol. 15, nº. 2, p. 193-205, 2018.

[9] KE, J.; ZHANG, D.; ZHENG, H.; An approximate dynamic programming approach to dynamic pricing for network revenue management. production and operations management, Vol. 28, $\mathrm{n}^{\circ}$. 11, p.2719-2737, 2019.

[10] CECHIN, R. B.; BIASUZ, R.; FAlAVIGNA, A.; CORSO, L. L.; Programação dinâmica aplicada à redução de custos nas compras de vacinas de um hospital. Medicina (Ribeirão Preto Online), Vol. 52, nº. 4, p.287-294, 2019.

[11] COLIN, E. C.; Pesquisa Operacional: 170 aplicações em estratégia, finanças, logística, produção, marketing e vendas. 2. Ed. [s.1]: Atlas, 2017.

[12] HILLIER, F. S.; LIEBERMAN, G. J.; Introdução à Pesquisa Operacional. 9. Ed. Porto Alegre: Amgh Editora Ltda, 2013.

[13] MARCONI, M. A.; LAKATOS, E. M.; Metodologia científica. 6. Ed. São Paulo: Atlas, 2011.

[14] DIBAN, P.; AZIZ, M. K. A.; FOO, D. C.y.; JIA, X.; LI, Z.; TAN, R. R.; Optimal biomass plantation replanting policy using dynamic programming. Journal of cleaner production, Vol. 126, $n^{\circ} 1, \mathrm{p}$ 409-418, 2016. 